\title{
Fibrolamellar carcinoma of the liver exhibits immunohistochemical evidence of both hepatocyte and bile duct differentiation
}

\author{
Stephen C Ward ${ }^{1,3}$, Jiaoti Huang ${ }^{1,4}$, Satish K Tickoo ${ }^{1}$, Swan N Thung ${ }^{2}$, Marc Ladanyi ${ }^{1}$ and \\ David S Klimstra ${ }^{1}$ \\ ${ }^{1}$ Department of Pathology, Memorial Sloan-Kettering Cancer Center, New York, NY, USA and ${ }^{2}$ The Lillian and \\ Henry M. Stratton-Hans Popper Department of Pathology, The Mount Sinai Medical Center, New York,
} $N Y, U S A$

Fibrolamellar carcinoma is a rare malignant primary liver neoplasm with characteristic histological features that typically arises in young patients without viral hepatitis or cirrhosis. Previous studies on this entity have been limited by small numbers of patients. In contrast to classical hepatocellular carcinoma, individual cases of fibrolamellar carcinoma have been reported to express cytokeratin 7 . In addition, ultrastructural and serological studies have suggested that fibrolamellar carcinoma may show neuroendocrine differentiation. The cellular differentiation of fibrolamellar carcinoma has not been studied and little is reported about its immunohistochemical profile. We studied 26 cases of fibrolamellar carcinoma and 62 cases of classical hepatocellular carcinoma by immunohistochemistry for HepPar1, glypican-3, pCEA, CD10, $\alpha$-fetoprotein, cytokeratin 20, neuroendocrine markers, and surrogate markers for biliary differentiation (cytokeratin 7, cytokeratin 19, epithelial membrane antigen, EpCAM, mCEA, B72.3, and CA19.9). In situ hybridization for albumin mRNA was also performed. Tumor cells of fibrolamellar carcinoma and hepatocellular carcinoma showed positive signals for albumin mRNA by in situ hybridization in all cases. Both tumor types stained uniformly positively with HepPar1 and most showed a canalicular staining pattern for pCEA, confirming their hepatocellular differentiation. In addition, $39 \%$ of hepatocellular carcinoma cases and $59 \%$ of fibrolamellar carcinoma cases were positive for glypican-3. All 22 fibrolamellar carcinoma cases tested showed positive staining for cytokeratin 7 and epithelial membrane antigen, whereas less than one-third of hepatocellular carcinoma cases were positive for these markers $(P<0.0001)$. Further, $36 \%$ of fibrolamellar carcinoma cases showed staining for B72.3, cytokeratin 19, EpCAM, or mCEA. Minimal evidence of neuroendocrine differentiation in either tumor was found with any of the usual immunohistochemical markers used for this purpose. Therefore, cytokeratin 7 and epithelial membrane antigen may be useful to differentiate between fibrolamellar carcinoma and hepatocellular carcinoma. On the basis of immunohistochemistry, fibrolamellar carcinoma seems to show both hepatocellular and bile duct differentiation.

Modern Pathology (2010) 23, 1180-1190; doi:10.1038/modpathol.2010.105; published online 21 May 2010

Keywords: albumin; fibrolamellar carcinoma; hepatocellular carcinoma; immunohistochemistry; in situ hybridization

Correspondence: Dr DS Klimstra, MD, Department of Pathology, Memorial Sloan-Kettering Cancer Center, 1275 York Avenue, New York, NY 10065, USA.

E-mail: klimstrd@mskcc.org

Portions of this study were presented at the 89th Annual Meeting of USCAP in New Orleans, LA, USA, March 2000.

${ }^{3}$ Current address: The Lillian and Henry M. Stratton-Hans Popper Department of Pathology, The Mount Sinai Medical Center, New York, NY, USA.

${ }^{4}$ Current address: Department of Pathology and Laboratory Medicine, UCLA Medical Center, Los Angeles, CA, USA.

Received 28 November 2009; revised 12 March 2010; accepted 15 March 2010; published online 21 May 2010
Fibrolamellar carcinoma is a rare primary liver tumor with a distinctive histology consisting of nest and cords of oncocytic cells with prominent nucleoli and abundant cytoplasm surrounded by lamellar arrays of collagen. ${ }^{1,2}$ Goodman et $a l^{3}$ also described a further variant of fibrolamellar carcinoma with mucinous differentiation in their description of combined hepatocellular cholangiocarcinomas. According to the Surveillance, Epidemiology and End Results (SEER) program, there were 153 cases of fibrolamellar carcinoma between 1990 and 2004 compared with 26503 cases of hepatocellular carcinoma, 4053 cases 
of cholangiocarcinoma, and 223 cases of mixed hepatocellular cholangiocarcinoma, ${ }^{4}$ which emphasizes the rarity of this neoplasm.

The clinical features of fibrolamellar carcinoma differ from both hepatocellular carcinoma and cholangiocarcinoma. Fibrolamellar carcinoma shows an even gender distribution, whereas the male to female ratio is 1.5:1 for cholangiocarcinoma and approximately 3:1 for hepatocellular carcinoma.,6 Fibrolamellar carcinoma is generally diagnosed in patients younger than 40 years, whereas hepatocellular carcinoma ${ }^{1,2,4,5,7}$ and cholangiocarcinoma ${ }^{6}$ usually affect patients $>60$ years of age. Unlike hepatocellular carcinoma, fibrolamellar carcinoma and cholangiocarcinoma are usually not associated with viral hepatitis or cirrhosis. ${ }^{1}$ Elevated serum levels of AFP are common in hepatocellular carcinoma but are not found in fibrolamellar carcinoma. Fibrolamellar carcinoma is reported to have a slightly better prognosis than classical hepatocellular carcinoma,, ,2,4,5,7-11 but this difference may be related to the younger age of fibrolamellar carcinoma patients ${ }^{12}$ and the lack of associated cirrhosis in fibrolamellar carcinoma. ${ }^{13}$

Although no true lineage-specific immunohistochemical markers have been identified for primary liver tumors, a panel of antibodies can be used to distinguish between hepatocellular carcinoma and cholangiocarcinoma. In general, hepatocellular carcinoma is positive for HepPar1, glypican-3, and AFP, and negative for CK7, CK 19, EMA, B72.3, mCEA, and CA19-9, whereas cholangiocarcinoma has the opposite staining characteristics. ${ }^{14-22}$ Hepatocellular carcinoma also shows a canalicular staining pattern with antibodies for pCEA and CD10, whereas cholangiocarcinoma shows either cytoplasmic or no staining with these antibodies. The immunohistochemical profile of fibrolamellar carcinoma has not been clearly defined. Most reports are limited by the number of cases, although some data suggest that fibrolamellar carcinoma stains for CK7, ${ }^{23-25}$ contrasting with conventional hepatocellular carcinoma.

Interestingly, patients with fibrolamellar carcinoma were reported to have increased levels of neurotensin in their serum. ${ }^{26}$ Ultrastructural studies have suggested that tumor cells of fibrolamellar carcinoma show neuroendocrine differentiation, ${ }^{27,28}$ although there has been no detailed immunohistochemical study to explore the frequency of neuroendocrine differentiation, relative to other primary hepatic carcinomas.

This study investigates the immunohistochemical and molecular profile of fibrolamellar carcinoma as well as the possibility of neuroendocrine differentiation of fibrolamellar carcinoma.

\section{Materials and methods}

\section{Selection of Cases}

A total of 40 specimens from 26 patients with fibrolamellar carcinoma and 62 specimens from 62 patients with hepatocellular carcinoma were retrieved from the files of the Department of Pathology, Memorial Sloan-Kettering Cancer Center, NY, USA, from 1988-2007. Identifying information was removed to protect patient confidentiality and the study was approved by the Memorial Sloan-Kettering Cancer Center internal review board. All 62 hepatocellular carcinoma specimens were primary resections. Several cases of fibrolamellar carcinoma had multiple resections within the archive. Specimens from primary tumors were available from 19 cases (12 cases with primary resections alone, 1 case with a biopsy from the primary tumor alone, 3 cases with primary resections and lymph node metastases, 2 cases with primary tumors and two distant metastatic sites each, and 1 case with the primary tumor, a liver recurrence, and a distant metastatic site). In one case, tissue was only available from a lymph node metastasis and in another three cases tissue was only available from distant metastatic sites. One case had tissue from a lymph node metastasis and three different distant metastatic sites, one case had tissue from a lymph node metastasis and one distant metastatic site, and one case had tissue from a liver recurrence and a lymph node metastasis. In addition, adjacent nonneoplastic liver tissue was analyzed from seven cases of fibrolamellar carcinoma. Hematoxylin and eosinstained slides from all the cases were reviewed and diagnoses were confirmed. Paraffin sections were prepared for in situ hybridization and immunohistochemistry.

\section{Tissue Microarrays}

Three $0.6-\mathrm{mm}$ cores of tumor tissue from 22 cases of fibrolamellar carcinoma and 52 cases of hepatocellular carcinoma and three cores from tissue adjacent to 7 cases of fibrolamellar carcinoma were assembled into three tissue microarrays. The tissue microarrays included five of the nine cases of fibrolamellar carcinoma and none of the ten cases of hepatocellular carcinoma used for whole-section staining.

\section{In Situ Hybridization}

In situ hybridization for albumin mRNA expression was performed using an antisense riboprobe hybridizing to a 322-bp portion of the albumin mRNA, consisting of nucleotides 163-484 (GenBank accession number V00495). Briefly, fresh-frozen normal human liver tissue was used for extraction of total RNA, from which the template for albumin riboprobe synthesis was generated by reverse-transcribed PCR (RT-PCR) (Ready-to-go RT-PCR beads; Pharmacia Biotech, Piscataway, NJ, USA). The first primer pair (T7-ALB-FWD-5'-AATTAATACGACTCACTAT AGAGGTTGCTCATCGGTTTA-3' and SP6-ALB-REV-A5'-CGATTTAGGTGACACTATAGGAGGTTTGGGTTGT 
Table 1 Antibodies used in immunohistochemistry

\begin{tabular}{lllll}
\hline Antibody & Company & Clonality & Dilution & Pretreatment \\
\hline AFP & Dako, Carpinteria, CO, USA & Polyclonal & $1: 1000$ & Sodium citrate (pH 6) \\
B72.3 & Biogenex, San Ramon, CA, USA & Monoclonal & $1: 500$ & Sodium citrate (pH 6) \\
CA19.9 & Signet, Dedham, MA, USA & Monoclonal & $1: 4000$ & Pepsin (0.05\%) \\
CD10 & Vector Laboratories, Burlingame, CA, USA & Monoclonal & $1: 50$ & Sodium citrate (pH 6) \\
mCEA & Dako & Monoclonal & $1: 500$ & Sodium citrate (pH 6) \\
pCEA & Dako & Polyclonal & $1: 6000$ & Trypsin (0.05\%) \\
Chromogranin & Dako & Polyclonal & $1: 8000$ & Sodium citrate (pH 6) \\
CK7 & Dako & Monoclonal & $1: 20000$ & Pronase (0.01\%) \\
CK20 & Dako & Monoclonal & $1: 1000$ & Protease (0.05\%) \\
CK19 & Dako & Monoclonal & $1: 1000$ & Sodium citrate (pH 6) \\
CD56 & Lab Vision, Fremont, CA, USA & Monoclonal & $1: 50$ & Sodium citrate (pH 6) \\
EMA & Dako & Monoclonal & $1: 100$ & None \\
EpCAM & Thermo Scientific, Waltham, MA, USA & Monoclonal & $1: 250$ & Antigen Unmasking Solution \\
& & & & (Vector Laboratories) \\
Glypican-3 & Biomosaics, Burlington, VT, USA & Monoclonal & $1: 250$ & Antigen Unmasking Solution \\
& & & (Vector Laboratories) \\
HepPar1 & Dako & Polyclonal & $1: 15000$ & Sodium citrate (pH 6) \\
Leu7 & Becton-Dickinson, San Jose, CA, USA & Monoclonal & $1: 50$ & Pronase (0.01\%) \\
NSE & Dako & Monoclonal & $1: 2000$ & Sodium citrate (pH 6) \\
p53 (DO-7) & Dako & Monoclonal & $1: 500$ & Sodium citrate (pH 6) \\
Synaptophysin & Dako & Polyclonal & $1: 500$ & Sodium citrate (pH 6) \\
& & &
\end{tabular}

CAT-3') included binding sites for T7 and SP6 RNA polymerases, respectively. The RT-PCR products were then reamplified using a second, outer primer pair (EcoRI-T7-5'-GCTTCGAATTCTAATACGACTC ACTATA-3' and BamHI-SP6-5'-ATACGGGATCCAT TTAGGTGACACTATA-3') (T7 and SP6 RNA polymerase binding sites) to add nucleotides to the ends of the PCR products for more efficient transcription and to introduce restriction endonuclease sites for plasmid cloning of the probe template, if necessary. Digoxigenin-labeled riboprobes (SP6ALB, antisense; T7-ALB, sense) were then generated by in vitro transcription using the appropriate RNA polymerase (DIG RNA-labeling kit; Boehringer Mannheim, Indianapolis, IN, USA). In situ hybridization was performed using the Dig-AP detection kit with NBT/BCIP as the chromogenic agent, according to the manufacturer's instructions (Zymed Laboratory, San Francisco, CA, USA). Distinct cytoplasmic bluish-black granularity was considered to be a positive result for albumin mRNA. Nonneoplastic liver and nonneoplastic biliary elements within the sections studied provided internal positive and negative controls, respectively. A sense sequence probe was also used as a negative control. Results were graded similarly to those for the keratin immunohistochemical stains.

\section{Immunohistochemistry}

Immunohistochemical staining was carried out on archival paraffin-embedded tissue sections using avidin-biotin complex technique. Briefly, 5- $\mu \mathrm{m}$ paraffin sections were prepared and mounted on
Superfrost Plus glass slides (Fisher Scientific, Pittsburgh, PA, USA). Deparaffinized sections were pretreated for antigen retrieval. Indirect immunoperoxidase staining was used according to standard protocols. The sections were incubated with a primary antibody followed by anti-mouse or rabbit immunoglobulin IgG conjugated with horseradish peroxidase. The peroxidase reactions were carried out using 3,3'-diaminobenzidine tetrahyhydrochloride (3,3'-diamonbenzidine). The sources of antibodies, dilutions, and method of antigen retrieval are detailed in Table 1.

The immunohistochemical staining was scored as: 1 (negative): no staining in any cells; 2 (focal positive): $<15 \%$ of tumor cells stained positively; and 3 (positive): $>15 \%$ of tumor cells stained positively. For pCEA and CD10 only a canalicular pattern of staining was scored as positive. The overall score was recorded across all cores from the same original tissue block present on the stained slide. At least one core from each tissue block must have been present on the stained slide for analysis.

\section{Statistics}

$\chi$-test was used to analyze differences in distribution and statistical significance was assigned as $P<0.05$. A case was considered positive for statistical analysis if any of the resection specimens from a patient showed positive staining in $>15 \%$ of the tumor cells. SPSS version 16.0 software (Chicago, IL, USA) was used for statistical analyses. 


\section{Results}

Clinical and Pathological Characteristics of Fibrolamellar Carcinoma and Hepatocellular Carcinoma Cases

The clinical characteristics of the 26 cases of fibrolamellar carcinoma and 62 cases of hepatocellular carcinoma are summarized in Table 2 . The mean age at diagnosis of fibrolamellar carcinoma was 25 years compared with 62.7 years for hepatocellular carcinoma. The male to female ratio for fibrolamellar carcinoma was 1:1.6, whereas the ratio for hepatocellular carcinoma was 4.2:1. In total, 13 of the 26 cases of fibrolamellar carcinoma had positive lymph nodes, whereas only one of 62 cases of hepatocellular carcinoma had lymph node involvement. None of the tested cases of fibrolamellar carcinoma were serologically positive for hepatitis B or $\mathrm{C}$ virus and none had elevated serum AFP ( $>15.0 \mathrm{ng} / \mathrm{ml}$ ), whereas 13 of 40 tested patients with hepatocellular carcinoma were positive for hepatitis B, 1 of 33 tested patients were positive for hepatitis C, and 19 of 39 cases had elevated serum AFP levels. The histological appearance of the fibrolamellar carcinoma cases was similar to that previously described, ${ }^{1,2}$ with nests and cords of large cells separated by variably dense bands of collagen arranged in parallel arrays. Rare pseudoglands were found in some cases. The nuclei were large and contained prominent nucleoli, and the cytoplasm was abundant, granular, and eosinophilic (Figure 1a). None of the cases showed mucinous differentiation as described by Goodman et al. ${ }^{3}$ The cases of hepatocellular carcinoma also showed typical histological features (Figure 1b).

\section{Fibrolamellar Carcinomas and Markers Associated with Hepatocellular Carcinoma}

Whole-section slides were stained for HepPar-1, pCEA, and AFP. As shown in Table 3, all cases of fibrolamellar carcinoma and hepatocellular carcinoma tested showed diffuse staining for HepPar1 and $80 \%$ of fibrolamellar carcinoma cases and $89 \%$ of hepatocellular carcinoma cases showed at least focal canalicular staining for pCEA. AFP was focally

Table 2 Clinical information

\begin{tabular}{lcc}
\hline & $\begin{array}{c}\text { Hepatocellular } \\
\text { carcinoma }\end{array}$ & $\begin{array}{c}\text { Fibrolamellar } \\
\text { carcinoma }\end{array}$ \\
\hline Age (years) & $62.7(26-84)$ & $25(14-48)$ \\
M:F & $4.2: 1$ & $1: 1.6$ \\
Size & $10.1(5.6)$ & $9.1(4.3)$ \\
$\%+L N$ & $1 / 62$ & $13 / 26$ \\
HepB+ & $13 / 40$ & $0 / 8$ \\
HepC+ & $1 / 33$ & $0 / 10$ \\
Elevated AFP & $19 / 39$ & $0 / 15$ \\
\hline
\end{tabular}

positive in $40 \%$ of hepatocellular carcinoma cases and negative in all fibrolamellar carcinoma cases. A positive signal was detected in all cases of fibrolamellar carcinoma and hepatocellular carcinoma tested by in situ hybridization with a probe specific for albumin mRNA (Table 3, Figure 1c and d). Tissue microarrays stained with glypican-3 showed that $59 \%$ of fibrolamellar carcinoma and $39 \%$ of hepatocellular carcinoma cases showed positive staining in $>15 \%$ of tumor cells (Figure 1e and f), whereas immunostaining for CD10 showed that $30 \%$ of hepatocellular carcinoma and $32 \%$ of fibrolamellar carcinoma cases demonstrated canalicular staining in at least $15 \%$ of tumor cells (Table 4). Positive nuclear staining for p53 was detected in at least 15\% of tumor cells in $38 \%$ of hepatocellular carcinoma cases and $23 \%$ of fibrolamellar carcinoma cases (Table 4).

\section{Fibrolamellar Carcinoma and Markers Associated with Cholangiocarcinoma}

Tissue microarrays were stained for CK7, EMA, B72.3, mCEA, CK19, EpCAM, and CA19-9, whereas whole slide sections were stained for CK20. Percentages of cases with at least one specimen showing positive ( $>15 \%$ of tumor cells) or focal positive $(<15 \%$ of tumor cells $)$ staining are shown in Table 4. CK7 and EMA showed positive staining in at least one specimen from all fibrolamellar carcinoma cases compared with less than one-third of hepatocellular carcinoma cases $(P<0.0001$; Figure 2a-d). B72.3 showed positive staining in $23 \%$ of fibrolamellar cases compared with $0 \%$ of hepatocellular carcinoma cases $(P<0.0001)$, although $2 \%$ of hepatocellular carcinoma cases showed focal positivity for this marker. Positive mCEA and EpCAM staining was seen in 9\% and $14 \%$ of fibrolamellar carcinoma cases, respectively, whereas no cases of hepatocellular carcinoma were positive for these markers $(P<0.01$; Figure $2 \mathrm{e}-\mathrm{f})$. CK19-positive staining was present in $23 \%$ of fibrolamellar carcinoma cases compared with $10 \%$ of hepatocellular carcinoma cases (not significant). In all, $36 \%$ of fibrolamellar carcinoma cases were positive for at least one of the above markers. Altogether, $2 \%$ of hepatocellular carcinoma cases were positive for CA19-9, whereas only focal staining was seen in any fibrolamellar carcinoma cases. Half of the hepatocellular carcinoma cases tested showed at least focal staining with CK20, whereas all fibrolamellar carcinoma cases tested were negative (Table 5).

The fibrolamellar carcinoma cases collected for study consisted of tissue from primary liver tumors (14 cases), liver recurrences (2 cases), lymph node metastases (6 cases), and distant metastases (9 cases). Multiple cases had tissue from more than one of these categories. The staining patterns were generally conserved over these categories (Table 4), 

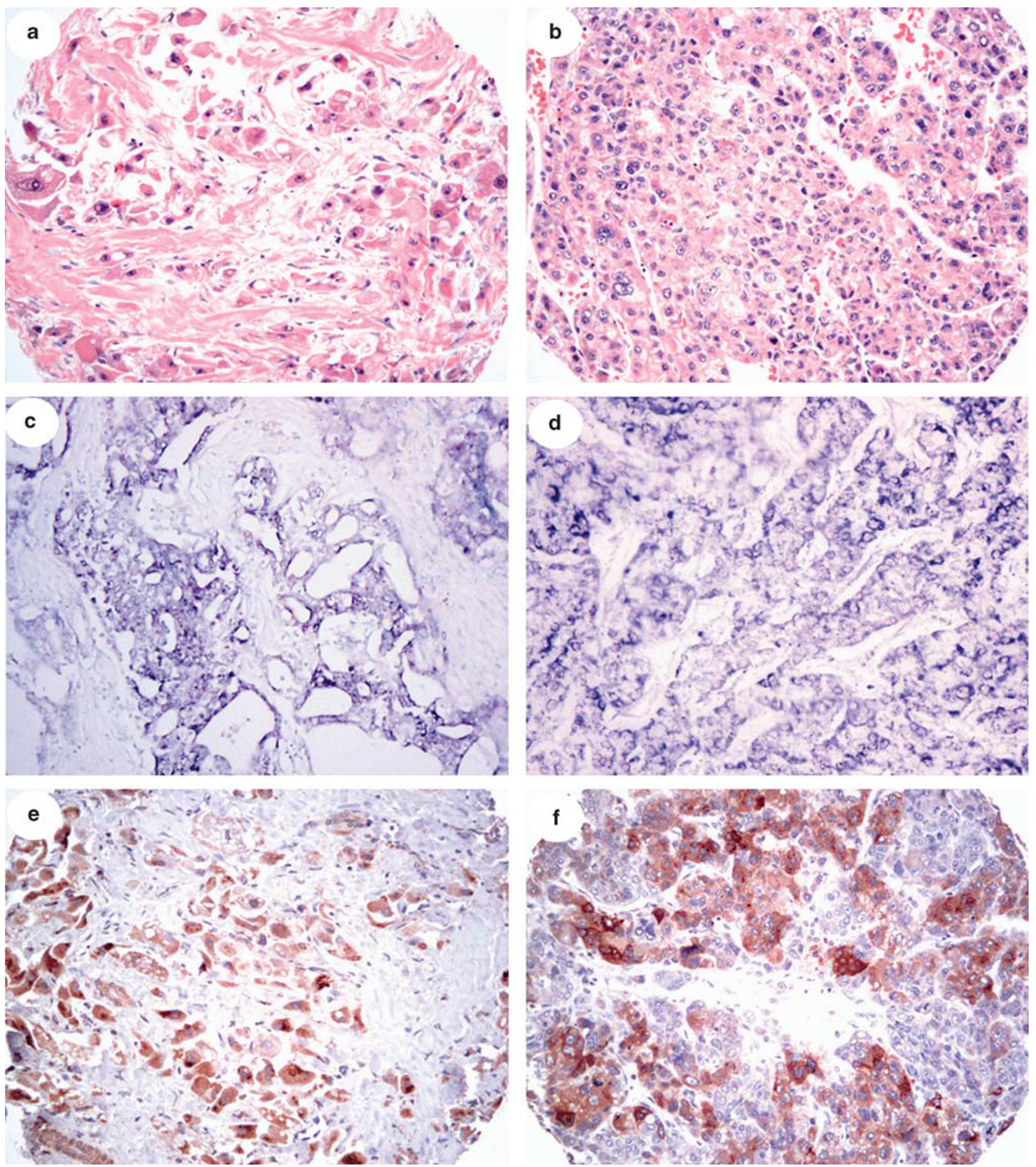

Figure 1 Examples of fibrolamellar carcinoma (a) and hepatocellular carcinoma (b) (hematoxylin and eosin, $\times 200)$. In situ hybridization using antisense probe for albumin mRNA was positive in all cases of fibrolamellar carcinoma (c) and hepatocellular carcinoma (d) tested $(\times 200)$. Immunohistochemical staining for glypican-3 was positive in cases of fibrolamellar carcinoma (e) and hepatocellular carcinoma (f) $(\times 200)$.

although there were only two cases in the liver recurrence group. The adjacent normal tissue from seven cases of fibrolamellar carcinoma all showed canalicular staining with CD10, whereas $29 \%$ showed positivity for CK7 and $43 \%$ showed positivity for EMA. The adjacent normal tissue was negative for p53, glypican-3, B72.3, mCEA, EpCAM, CK19, and CA19-9 (Table 4). 
Table 3 Results of immunohistochemical stains with pCEA, HepPar1, and anti-AFP, and in situ hybridization with an albumin mRNA probe

\begin{tabular}{|c|c|c|c|c|c|c|}
\hline & \multirow[b]{2}{*}{$\mathrm{n}$} & \multirow{2}{*}{$\begin{array}{c}\text { HepPar } \\
\% \text { Positive (\% FP) }\end{array}$} & \multirow{2}{*}{$\begin{array}{c}p C E A^{\mathrm{a}} \\
\% \text { Positive }(\% \mathrm{FP})\end{array}$} & \multirow{2}{*}{$\begin{array}{c}A F P \\
\% \text { Positive }(\% F P)\end{array}$} & \multicolumn{2}{|c|}{ Albumin mRNA probe } \\
\hline & & & & & Anti-sense & Sense \\
\hline Hepatocellular carcinoma & 10 & $100(0)$ & $0(80)$ & $0(40)$ & 100 & 0 \\
\hline Fibrolamellar carcinoma & 9 & $100(0)$ & $11(78)$ & $0(0)$ & 100 & 0 \\
\hline
\end{tabular}

${ }^{\mathrm{a} O n l y}$ canalicular staining pattern is considered true staining.

Table 4 Results of immunohistochemical stains for p53, glypican-3, and markers associated with biliary differentiation

\begin{tabular}{|c|c|c|c|c|c|c|c|c|c|c|c|}
\hline & $\mathrm{n}$ & $\begin{array}{c}p 53 \\
\% \text { Positive } \\
(\% \text { FP) }\end{array}$ & $\begin{array}{c}\text { Glypican-3 } \\
\% \text { Positive } \\
(\% \text { FP) }\end{array}$ & $\begin{array}{c}\text { CD10 } \\
\% \text { Positive } \\
(\% \text { FP })\end{array}$ & $\begin{array}{c}\text { CK7 } \\
\% \text { Positive } \\
(\% \text { FP })\end{array}$ & $\begin{array}{c}\text { EMA } \\
\% \text { Positive } \\
(\% \text { FP) }\end{array}$ & $\begin{array}{c}\text { B72.3 } \\
\% \text { Positive } \\
(\% \text { FP })\end{array}$ & $\begin{array}{c}\text { mCEA } \\
\% \text { Positive } \\
(\% \text { FP })\end{array}$ & $\begin{array}{c}\text { EpCAM } \\
\% \text { Positive } \\
(\% \text { FP) }\end{array}$ & $\begin{array}{c}\text { CK19 } \\
\% \text { Positive } \\
(\% F P)\end{array}$ & $\begin{array}{c}\text { CA19-9 } \\
\% \text { Positive } \\
(\% \text { FP })\end{array}$ \\
\hline HCC & 50 & $38(18)$ & $39(0)^{\mathrm{a}}$ & $30(6)$ & $28(32)$ & $28(6)$ & $0(2)$ & $0(0)$ & $0(0)^{\mathrm{a}}$ & $10(4)$ & $2(4)$ \\
\hline FLC & 22 & 23 (14) & $59(0)$ & $32(5)$ & $100(0)$ & $100(0)$ & $23(23)$ & $9(0)$ & $14(5)$ & $23(0)$ & $0(9)$ \\
\hline$P$-value & & NS & NS & NS & $<0.0001$ & $<0.0001$ & $<0.0001$ & $<0.01$ & $<0.01$ & NS & NS \\
\hline FLC $1^{\circ}$ & 14 & $7(7)$ & $64(0)$ & $21(7)$ & $100(0)$ & $93(0)$ & $14(21)$ & $14(0)$ & $14(0)$ & $21(0)$ & $0(7)$ \\
\hline FLC recur. & 2 & $0(0)$ & $100(0)$ & $0(0)$ & $100(0)$ & $100(0)$ & $0(50)$ & $0(0)$ & $0(0)$ & $0(0)$ & $0(0)$ \\
\hline FLC LN & 6 & $40(20)^{\mathrm{b}}$ & $33(0)$ & $20(0)^{\mathrm{b}}$ & $100(0)$ & $100(0)$ & 33 (17) & $17(0)$ & $20(20)^{\mathrm{b}}$ & $33(0)$ & $0(0)^{\mathrm{b}}$ \\
\hline FLC met. & 9 & $44(22)$ & $78(0)$ & $33(0)$ & $100(0)$ & $100(0)$ & $22(22)$ & $0(0)$ & $11(0)$ & $11(0)$ & 0 (11) \\
\hline Normal & 7 & 0 (14) & $0(0)$ & $100(0)$ & $29(57)$ & $43(0)$ & $0(0)$ & $0(0)$ & $0(0)$ & $0(0)$ & $0(0)$ \\
\hline
\end{tabular}

HCC, hepatocellular carcinoma; FLC, fibrolamellar carcinoma; $1^{\circ}$, primary tumor; recur., local recurrence; LN, lymph node metastasis; met., distant metastasis.

${ }^{\mathrm{a}} N=51$.

${ }^{\mathrm{b}} N=5$.

\section{Fibrolamellar Carcinoma Rarely Shows Neuroendocrine Differentiation by Immunohistochemistry}

Whole slide sections were stained for chromogranin, synaptophysin, NSE, CD56, and Leu7. As shown in Table 5, most cases of fibrolamellar carcinoma and hepatocellular carcinoma tested were negative for all the neuroendocrine markers studied except CD56. Only rare cases of fibrolamellar carcinoma and hepatocellular carcinoma showed focal positivity for one or two of the other markers. The most specific markers (chromogranin and synaptophysin) were only focally expressed in one case of hepatocellular carcinoma, whereas all fibrolamellar carcinomas were negative for these markers. Fibrolamellar carcinomas did not show more frequent or widespread neuroendocrine differentiation than hepatocellular carcinomas.

\section{Discussion}

Fibrolamellar carcinoma is a rare primary liver tumor with distinctive histological and clinical features separating it from both hepatocellular carcinoma and cholangiocarcinoma. Although the morphological features of fibrolamellar carcinoma strongly suggest that it shows hepatocellular differentiation, there are sufficient differences from classical hepatocellular carcinoma in the association with hepatitis and cirrhosis and in the production of AFP to raise questions about the cellular differentiation of fibrolamellar carcinoma. The occurrence of a mucin-producing variant of fibrolamellar carcinoma ${ }^{3}$ raises the possibility of bile duct differentiation in this entity, although mucin production seems to be a rare feature. The current study was carried out to clarify the immunophenotype of this rare hepatic neoplasm. The cases included in this study represented pathologically and clinically typical examples of the fibrolamellar carcinoma and did not include examples of the mucin-producing variant of fibrolamellar carcinoma.

Although there are no lineage-specific immunohistochemical markers for hepatocellular carcinoma or cholangiocarcinoma, a panel of antibodies can be used to distinguish between these tumors. HepPar1 is a monoclonal antibody that specifically reacts with hepatocytes, ${ }^{29}$ although some tumors other than hepatocellular carcinoma may stain (eg, adenocarcinomas of the stomach, ovary, and lung, and intraductal oncocytic papillary neoplasms of the pancreas and liver).$^{30}$ Glypican-3 is a heparin sulfate proteoglycan that is expressed in fetal liver and in $\sim 80 \%$ of hepatocellular carcinoma cases. ${ }^{21,22}$ AFP is a rather specific but relatively insensitive immunohistochemical marker of hepatocellular carcinoma ${ }^{33-35}$ although marked serum elevations of 

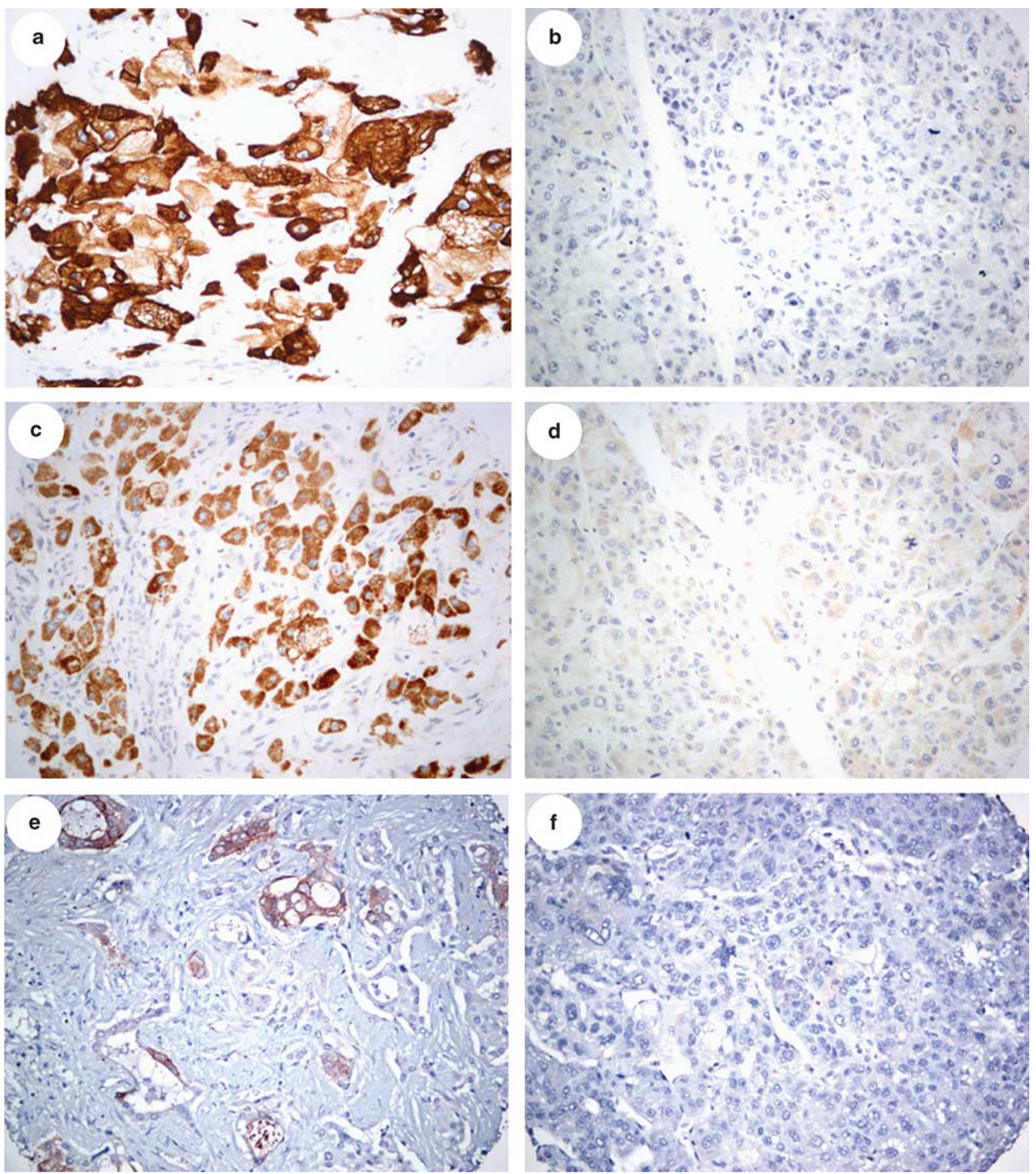

Figure 2 Immunohistochemical stains for CK7 and EMA were strongly positive in all cases of fibrolamellar carcinoma (a and c, respectively), whereas most cases of hepatocellular carcinoma were negative for CK7 and EMA (b and d, respectively) ( $\times 200)$. Immunohistochemical stains for EpCAM showed that $14 \%$ of fibrolamellar carcinoma cases were positive (e), whereas no cases of hepatocellular carcinoma were positive $(\mathbf{f})(\times 200)$.

AFP are reasonably predictive of the diagnosis. The polyclonal CEA antibody stains normal liver and hepatocellular carcinoma in a characteristic canalicular pattern because of its cross-reaction with biliary glycoprotein $\mathrm{I}^{31-35} \mathrm{CD} 10$ also has the same staining pattern as pCEA. ${ }^{36}$ Probably, the most specific test to document hepatocellular differentiation is in situ hybridization for albumin, which is reported in be positive in $75-96 \%$ of hepatocellular carcinoma and can also be useful to demonstrate 
Table 5 Results of immunohistochemical stains for CK20 and markers associated with neuroendocrine differentiation

\begin{tabular}{|c|c|c|c|c|c|c|c|}
\hline & $\mathrm{n}$ & $\begin{array}{c}\text { CK20 } \\
\% \text { Positive } \\
(\% \text { FP) }\end{array}$ & $\begin{array}{c}\text { SYN } \\
\% \text { Positive } \\
(\% \text { FP) }\end{array}$ & $\begin{array}{c}\text { Chr } \\
\% \text { Positive } \\
(\% \text { FP) }\end{array}$ & $\begin{array}{c}\text { Leu7 } \\
\% \text { Positive } \\
(\% \text { FP) }\end{array}$ & $\begin{array}{c}\text { CD56 } \\
\% \text { Positive } \\
(\% \text { FP })\end{array}$ & $\begin{array}{c}\text { NSE } \\
\% \text { Positive } \\
(\% \text { FP) }\end{array}$ \\
\hline Hepatocellular carcinoma & 10 & $30(20)$ & $0(10)$ & $0(10)$ & $0(20)$ & $0(30)$ & $0(0)$ \\
\hline Fibrolamellar carcinoma & 9 & $0(0)$ & $0(0)$ & $0(0)$ & $0(0)$ & $33(56)$ & $0(11)$ \\
\hline
\end{tabular}

hepatocellular differentiation in combined hepatocellular cholangiocarcinomas. ${ }^{37-39}$ The immunohistochemical profile for cholangiocarcinoma is less distinctive. HepPar1 and AFP staining have not been reported in cases of cholangiocarcinoma, and these cases do not show a canalicular pattern of staining with pCEA or CD10. The majority of cases of cholangiocarcinoma, however, stain for CK7, ${ }^{15-19}$ $\mathrm{CK} 19,{ }^{15-18}$ and EMA,${ }^{20}$ and there may be staining for the glycoprotein markers B72.3, mCEA, ${ }^{19}$ and CA19-9. ${ }^{20}$ Although these antibodies are not specific for cholangiocarcinoma, they are rarely positive in hepatocellular carcinoma.

We applied the above panel of antibodies to fibrolamellar carcinoma and found that all cases tested showed staining with HepPar1 and had albumin mRNA detectable by in situ hybridization. We showed that $59 \%$ of fibrolamellar carcinoma cases were positive for glypican-3, consistent with the findings of Shafizadeh et al. ${ }^{22}$ However, we found that only $39 \%$ of our hepatocellular carcinoma cases were positive for glypican-3, much lower than the $70-100 \%$ range reported by Shafizadeh et $a 1^{22}$ and other groups. ${ }^{21,40-43}$ Our study used a tissue microarray for glypican-3 immunostaining in contrast to whole-section staining performed in the other studies; thus, the possibility of focal staining elsewhere in the tumors cannot be excluded.

Fibrolamellar carcinomas also strongly expressed some markers more typically associated with bile duct differentiation (EMA and CK7). In addition, over a third of the cases of fibrolamellar carcinoma also stained for B72.3, CK19, EpCAM, or mCEA, which are biliary markers very rarely seen in cases of hepatocellular carcinoma. Although some of these markers were also expressed in a small subset of classical hepatocellular carcinomas (see Table 4), there was significantly more labeling of fibrolamellar carcinomas than hepatocellular carcinomas for most bile duct-associated markers. EpCAM and CK19 positivity have also been associated with worse prognosis in hepatocellular carcinoma, possibly related to a progenitor cell phenotype. ${ }^{44-48}$ Interestingly, hepatoblastomas ${ }^{49,50}$ and hepatocellular carcinomas occurring in pediatric patients also show increased staining for biliary and progenitor cell markers. ${ }^{51}$ The increased staining of fibrolamellar carcinomas with EpCAM may indicate that these tumors have a progenitor cell phenotype with both hepatocellular and biliary differentiation or may be related to the development of these tumors at a younger age.

Cholangiocarcinoma can be further subdivided by location into peripheral, hilar, and extrahepatic. The immunohistochemical profiles of these subdivisions have recently been investigated, showing that the peripheral cholangiocarcinomas show less staining with glycoprotein markers such as B72.3. In this regard, fibrolamellar carcinomas appear to most resemble peripheral cholangiocarcinomas (rather than hilar or extrahepatic) immunohistochemically.

In our study, $40 \%$ of hepatocellular carcinomas were focally positive for AFP, which is consistent with previous reports. Unlike patients with hepatocellular carcinoma who often have increased serum levels of AFP, serum AFP levels are normal in patients with fibrolamellar carcinoma, ${ }^{35}$ and none of the nine fibrolamellar carcinoma cases that we tested was positive for AFP by immunohistochemistry.

As expected, the adjacent nonneoplastic tissue showed canalicular staining with CD10 in all the seven cases tested. Many of these cases also showed positivity for CK7 and EMA. This may indicate a reactive upregulation of biliary-type markers in the liver tissue adjacent to tumors, as is seen in response to other liver injuries or diseases..52,53

None of the nine cases of fibrolamellar carcinoma tested were positive for CK20. However, we showed that three of ten cases of hepatocellular carcinoma were positive for CK20, whereas an additional two cases were focally positive. Leung et $a l^{54}$ showed that only 2 of 32 excisional or needle biopsies of hepatocellular carcinoma were positive for CK20, whereas Saleh et $a 1^{55}$ found that 6 of 42 fine needle aspirate specimens from hepatocellular carcinoma were positive for CK20. Our higher CK20 positivity rate in hepatocellular carcinoma may be due to our use of tissue from resection specimens, although conclusions drawn from our results are limited by the small sample size tested.

Previously, it was proposed that fibrolamellar carcinoma may show neuroendocrine differentiation based largely on ultrastructural studies. The cytoplasm of the tumor cells was found to have uranaffin-positive dense-core secretory granules. ${ }^{11}$ This finding was in keeping with the fact that patients with fibrolamellar carcinomas often have increased serum levels of neurotensin. ${ }^{10}$ However, there has not been any previous report to corroborate 
these findings with immunohistochemical studies using the commonly used specific neuroendocrine markers. The potential for some degree of neuroendocrine differentiation in hepatocellular neoplasms may not be surprising. Studies have shown the possible roles of neuroendocrine cells in hepatitis B virus-associated hepatocellular carcinoma, ${ }^{56}$ focal nodular hyperplasia, ${ }^{57}$ and precursor lesions of hepatocellular carcinoma, including small cell change ${ }^{58}$ dysplastic nodules, ${ }^{59}$ hepatic adenoma ${ }^{60}$ and reactive bile ductules. ${ }^{61}$ Furthermore, some degree of neuroendocrine differentiation has been previously reported in hepatocellular carcinomas. Wang et $a l^{62}$ studied 16 cases of hepatocellular carcinoma with neuroendocrine markers NSE, protein gene product 9.5, vasoactive intestinal polypeptide, calcitonin, and the Grimelius stain. In all, 11 cases were positive for protein gene product 9.5, 4 for NSE, 8 for vasoactive intestinal peptide, 2 for calcitonin, and 7 showed granular staining by the Grimelius method. Zhao et $a l^{63}$ used a large battery of general and specific neuroendocrine markers to study hepatocellular carcinomas from 50 patients. They found that $60 \%$ of their cases were positive for one or more neuroendocrine markers and multireactivity was noted in $24 \%$ of the cases. In this study, we found minimal evidence of neuroendocrine differentiation in fibrolamellar carcinoma by staining for synaptophysin, chromogranin, NSE, CD56, and Leu7. Several cases of hepatocellular carcinoma and fibrolamellar carcinoma showed at least focal staining for CD56 (30 and 89\%, respectively). The positive staining of fibrolamellar carcinoma for CD56 may be a further evidence of ductular differentiation, ${ }^{61}$ although the predominantly focal-staining pattern and lack of staining with other neuroendocrine markers suggest that the CD56 staining may be nonspecific. As we used only five of the most commonly used general neuroendocrine markers, it would not be surprising if a more extensive battery of specific neuroendocrine markers may detect reactivity in more cases. But our data suggest that fibrolamellar carcinoma is not fundamentally a neuroendocrine tumor and probably has no more potential for minor neuroendocrine differentiation than hepatocellular carcinoma.

We demonstrated an increased incidence of lymph node metastasis in fibrolamellar carcinoma compared with hepatocellular carcinoma, as has been described in previous reports. ${ }^{1,64,65}$ Half of our cases of fibrolamellar carcinoma had lymph node metastases compared with only 1 of 62 cases of hepatocellular carcinoma. Cholangiocarcinomas ${ }^{66,67}$ and hepatoblastomas ${ }^{68}$ also show higher rates of lymph node metastasis at surgery than hepatocellular carcinoma, suggesting a link between these tumors and fibrolamellar carcinomas. However, as our study and many others only include patients who underwent surgical resection, this does not accurately represent the rate of lymph node metastasis at presentation for these various tumors. Most cases of hepatocellular carcinoma with lymph node metastasis are deemed inoperable, whereas resections are still performed for patients with fibrolamellar carcinoma, cholangiocarcinoma and hepatoblastoma with extrahepatic spread.

In summary, fibrolamellar carcinoma affects a younger patient population than both hepatocellular carcinoma and cholangiocarcinoma. Fibrolamellar carcinoma histologically resembles hepatocellular carcinoma and the albumin mRNA in situ hybridization assay demonstrates that these tumors show hepatocellular differentiation. Immunohistochemically, however, fibrolamellar carcinomas share features with both hepatocellular carcinoma and cholangiocarcinoma. The uniformity of staining with HepPar1, CK7, and EMA show that these are true markers of fibrolamellar carcinoma, rather than random alterations in the makeup of these tumors. In addition, $36 \%$ of fibrolamellar carcinoma cases show staining for B72.3, CK19, EpCAM, or mCEA, further demonstrating their relationship with cholangiocarcinoma. As most of the immunohistochemical stains in this study were performed on tissue microarrays, our results may actually underestimate the immunohistochemical evidence of biliary differentiation in fibrolamellar carcinoma. Our findings suggest that fibrolamellar carcinoma could be derived from a precursor cell with the ability to differentiate into hepatocytes or bile duct cells. Recognition of this distinctive immunophenotypes may aid in the diagnostic evaluation of primary hepatic neoplasms.

\section{Disclosure/conflict of interest}

The authors declare no conflict of interest.

\section{References}

1 Craig JR, Peters RL, Edmondson HA, et al. Fibrolamellar carcinoma of the liver: a tumor of adolescents and young adults with distinctive clinico-pathologic features. Cancer 1980;46:372-379.

2 Berman MM, Libbey NP, Foster JH. Hepatocellular carcinoma polygonal cell type with fibrous stroma-an atypical variant with favorable prognosis. Cancer 1980; 46:1448-1455.

3 Goodman ZD, Ishak KG, Langloss JM, et al. Combined hepatocellular-cholangiocarcinoma a histologic and immunohistochemical study. Cancer 1985;55:124-135.

4 Surveillance, Epidemiology, and End Results (SEER) Program (www.seer.cancer.gov) SEER*Stat Database: Incidence-SEER 17 Regs Limited-Use, November 2006 Sub (1973-2004 varying)—Linked To County Attributes-Total US, 1969-2004 Counties, National Cancer Institute, DCCPS, Surveillance Research Program, Cancer Statistics Branch, released April 2007, based on the November 2006 submission.

5 El-Serag HB, Davila JA. Is fibrolamellar carcinoma different from hepatocellular carcinoma? A US population-based study. Hepatology 2004;39:798-803. 
6 Lazaridis KN, Gores GJ. Cholangiocarcinoma. Gastroenterology 2005;128:1655-1667.

7 Stipa F, Yoon SS, Liau KH, et al. Outcome of patients with fibrolamellar hepatocellular carcinoma. Cancer 2006;106:1331-1338.

8 Berman MM, Libbey NP, Foster JH. Hepatocellular carcinoma. Polygonal cell type with fibrous stromaan atypical variant with a favorable prognosis. Cancer 1980;46:1448-1455.

9 Nagorney DM, Adson MA, Weiland LH, et al. Fibrolamellar hepatoma. Am J Surg 1985;149:113-119.

10 Epstein BE, Pajak TF, Haulk TL, et al. Metastatic nonresectable fibrolamellar hepatoma: prognostic features and natural history. Am J Clin Oncol 1999;22:22-28.

11 El-Gazzaz G, Wong W, El-Hadary MK, et al. Outcome of liver resection and transplantation for fibrolamellar hepatocellular carcinoma. Transpl Int 2000;13(Suppl 1): S406-S409.

12 Katzenstein HM, Krailo MD, Malogolowkin MH, et al. Fibrolamellar hepatocellular carcinoma in children and adolescents. Cancer 2003;97:2006-2012.

13 Kakar S, Burgart LJ, Batts KP, et al. Clinicopathologic features and survival in fibrolamellar carcinoma: comparison with conventional hepatocellular carcinoma with and without cirrhosis. Mod Pathol 2005;18: 1417-1423.

14 Tickoo SK, Zee SY, Obiekwe S, et al. Combined hepatocellular-cholangiocarcinoma: a histopathologic, immunohistochemical, and in situ hybridization study. Am J Surg Pathol 2002;26:989-997.

15 Stroescu C, Herlea V, Dragnea A, et al. The diagnostic value of cytokeratins and carcinoembryonic antigen immunostaining in differentiating hepatocellular carcinomas from intrahepatic cholangiocarcinomas. J Gastrointestin Liver Dis 2006;15:9-14.

16 Maeda T, Kajiyama K, Adachi E, et al. The expression of cytokeratins 7,19 , and 20 in primary and metastatic carcinomas of the liver. Mod Pathol 1996;9:901-909.

17 Lai YS, Thung SN, Gerber MA, et al. Expression of cytokeratins in normal and diseased livers and in primary liver carcinomas. Arch Pathol Lab Med 1989; 113:134-138.

18 D'Errico A, Baccarini P, Fiorentino M, et al. Histogenesis of primary liver carcinomas: strengths and weaknesses of cytokeratin profile and albumin mRNA detection. Hum Pathol 1996;27:599-604.

19 Lau SK, Prakash S, Geller SA, et al. Comparative immunohistochemical profile of hepatocellular carcinoma, cholangiocarcinoma, and metastatic adenocarcinoma. Hum Pathol 2002;33:1175-1181.

20 Tsuji M, Kashihara T, Terada N, et al. An immunohistochemical study of hepatic atypical adenomatous hyperplasia, hepatocellular carcinoma, and cholangiocarcinoma with alpha-fetoprotein, carcinoembryonic antigen, CA19-9, epithelial membrane antigen, and cytokeratins 18 and 19. Pathol Int 1999;44:310-317.

21 Nakatsura T, Yoshitake Y, Senju S, et al. Glypican-3, overexpressed specifically in human hepatocellular carcinoma, is a novel tumor marker. Biochem Biophys Res Commun 2003;306:16-25.

22 Shafizadeh N, Ferrell LD, Kakar S. Utility and limitations of glypican-3 expression for the diagnosis of hepatocellular carcinoma at both ends of the differentiation spectrum. Mod Pathol 2008;21:1011-1018.

23 Van Eyken P, Sciot R, Brock P, et al. Abundant expression of cytokeratin 7 in fibrolamellar carcinoma of the liver. Histopathology 1990;17:101-107.
24 Gornicka B, Ziarkiewicz-Wroblewska B, Wroblewski T, et al. Carcinoma, a fibrolamellar variant-immunohistochemical analysis of 4 cases. Hepatogastroenterology 2005;52:519-523.

25 Klein WM, Molmenti EP, Colombani PM, et al. Primary liver carcinoma arising in people younger than 30 years. Am J Clin Pathol 2005;124:491-493.

26 Collier NA, Weinbren K, Bloom SR, et al. Neurotensin secretion by fibrolamellar carcinoma of the liver. Lancet 1984;323:538-540.

27 Payne CM, Nagle RB, Paplanus SH, et al. Fibrolamellar carcinoma of liver: a primary malignant oncocytic carcinoid? Ultrastruct Pathol 1986;10:539-552.

28 Lloreta J, Vadell C, Fabregat X, et al. Fibrolamellar hepatic tumor with neurosecretory features and systemic deposition of AA amyloid. Ultrastruct Pathol 1994;18:287-292.

29 Wennerberg AE, Nalesnik MA, Coleman WB. Hepatocyte paraffin 1: a monoclonal antibody that reacts with hepatocytes and can be used for differential diagnosis of hepatic tumors. Am J Pathol 1993;143:1050-1054.

30 Chu PG, Ishizawa S, Wu E, et al. Hepatocyte antigen as a marker of hepatocellular carcinoma: an immunohistochemical comparison to carcinoembryonic antigen, CD10, and alpha-fetoprotein. Am J Surg Pathol 2002;26:978-988.

31 Christensen WN, Boitnott JK, Kuhajda FP. Immunoperoxidase staining as a diagnostic aid for hepatocellular carcinoma. Mod Pathol 1989;2:8-12.

32 Roncalli M, Borzio M, de Biagi G, et al. Liver cell dysplasia and hepatocellular carcinoma: a histological and immunohistochemical study. Histopathology 1985;9:209-921.

33 Koelma IA, Nap M, Huitema S, et al. Hepatocellular carcinoma, adenoma, and focal nodular hyperplasia. Comparative histopathologic study with immunohistochemical parameters. Arch Pathol Lab Med 1986;110:1035-1040.

34 Machinami R, Oono Y. Carcinoembryonic antigen and lectin binding in the bile canalicular structures of hepatocellular carcinoma. Virchows Arch A Pathol Anat Histopathol 1987;412:111-118.

35 Berman MA, Burnham JA, Sheahan DG. Fibrolamellar carcinoma of the liver: an immunohistochemical study of nineteen cases and a review of the literature. Hum Pathol 1988;19:784-794.

36 Chu P, Arber DA. Paraffin-section detection of CD10 in 505 nonhematopoietic neoplasms. Frequent expression in renal cell carcinoma and endometrial stromal sarcoma. Am J Clin Pathol 2000;113:374-382.

37 Murray GI, Paterson PJ, Ewen SW, et al. In situ hybridisation of albumin mRNA in normal liver and hepatocellular carcinoma with a digoxigenin labelled oligonucleotide probe. J Clin Pathol 1992;45: 21-24.

38 Yamaguchi K, Nalesnik MA, Carr BI. In situ hybridization of albumin mRNA in normal liver and liver tumors: identification of hepatocellular origin. Virchows Arch B Cell Pathol Incl Mol Pathol 1993;64: 361-365.

39 Krishna M, Lloyd RV, Batts KP. Detection of albumin messenger RNA in hepatic and extrahepatic neoplasms. A marker of hepatocellular differentiation. Am J Surg Pathol 1997;21:147-152.

40 Capurro M, Wanless IR, Sherman M, et al. Glypican-3: a novel serum and histochemical marker for hepatocellular carcinoma. Gastroenterology 2003;125:89-97. 
41 Yamauchi N, Watanabe A, Hishinuma M, et al. The glypican 3 oncofetal protein is a promising diagnostic marker for hepatocellular carcinoma. Mod Pathol 2005;18:1591-1598.

42 Wang XY, Degos F, Dubois S, et al. Glypican-3 expression in hepatocellular tumors: diagnostic value for preneoplastic lesions and hepatocellular carcinomas. Hum Pathol 2006;37:1435-1441.

43 Libbrecht L, Severi T, Cassiman D, et al. Glypican-3 expression distinguishes small hepatocellular carcinomas from cirrhosis, dysplastic nodules, and focal nodular hyperplasia-like nodules. Am J Surg Pathol 2006;30:1405-1411.

44 Durnez A, Verslype C, Nevens F, et al. The clinicopathological and prognostic relevance of cytokeratin7 and 19 expression in hepatocellular carcinoma. A possible progenitor cell origin. Histopathology 2006; 49:138-151.

45 Yang XR, Xu Y, Shi GM, et al. Cytokeratin 10 and cytokeratin 19: predictive markers for poor prognosis in HCC patients after curative resection. Clin Cancer Res 2008;14:3850-3859.

46 Zhuang PY, Zhang JB, Zhu XD, et al. Two pathologic types of hepatocellular carcinoma with lymphnode metastasis with distinct prognosis on the basis of CK19 expression in tumor. Cancer 2008;112:2740-2748.

47 Trzpis M, McLaughlin PMJ, de Leij LM, et al. Epithelial cell adhesion molecule. More than a carcinoma marker and adhesion molecule. Am J Pathol 2007;171:386-395.

48 Yamashita T, Forgues M, Wang W, et al. EpCAM and alpha-fetoprotein expression defines novel prognostic types of HCC. Cancer Res 2008;68:1451-1461.

49 Ruck P, Xiao JC, Pietsch T, et al. Hepatic stem-like cells in hepatoblastoma: expression of cytokeratin 7, albumin and oval cell associated antigens detected by OV-1 and OV-6. Histopathology 1997;31:324-329.

50 Ruck P, Wichert G, Handgretinger R, et al. Ep-CAM in malignant liver tumours. J Pathol 2000;191: 102-103.

51 Ward SC, Thung SN, Lim KH, et al. Hepatic progenitor cells in liver cancers from Asian children. Liver Int 2010;30:102-111.

52 Katoonizadeh A, Nevens F, Verslype C, et al. Liver regeneration in acute severe liver impairment: a clinicopathological correlation study. Liver Int 2006; 26:1225-1233.

53 Tan J, Hytiroglou P, Wieczorek R, et al. Immunohistochemical evidence for hepatic progenitor cells in liver diseases. Liver 2002;22:365-373.

54 Leong AS, Sormunen RT, Tsui WM, et al. Hep Par 1 and selected antibodies in the immunohistological distinction of hepatocellular carcinoma from cholangiocarcinoma, combined tumours and metastatic carcinoma. Histopathology 1998;33:318-324.
55 Saleh HA, Aulicino M, Zaidi SY, et al. Discriminating hepatocellular carcinoma from metastatic carcinoma on fine-needle aspiration biopsy of the liver: the utility of immunocytochemical panel. Diagn Cytopathol 2009;37:184-190.

56 Hsia CC, Evarts RP, Nakatsukasa H, et al. Occurrence of oval-type cells in hepatitis B virus-associated human hepatocarcinogenesis. Hepatology 1992;16:1327-1333.

57 Roskams T, De Vos R, Desmet V. 'Undifferentiated progenitor cells' in focal nodular hyperplasia of the liver. Histopathology 1996;28:291-299.

58 Libbrecht L, Desmet V, Van Damme B, et al. The immunohistochemical phenotype of dysplastic foci in human liver: correlation with putative progenitor cells. J Hepatol 2000;33:76-84.

59 Le Bail B, Faouzi S, Boussarie L, et al. Extracellular matrix composition and integrin expression in early hepatocarcinogenesis in human cirrhotic liver. J Pathol 1997;181:330-337.

60 Libbrecht L, De Vos R, Cassiman D, et al. Hepatic progenitor cells in hepatocellular adenomas. Am J Surg Pathol 2001;25:1388-1396.

61 Roskams T, van den Oord JJ, De Vos R, et al. Neuroendocrine features of reactive bile ductules in cholestatic liver disease. Am J Pathol 1990;137: 1019-1025.

62 Wang JH, Dhillon AP, Sankey EA, et al. 'Neuroendocrine' differentiation in primary neoplasms of the liver. J Pathol 1991;163:61-67.

63 Zhao M, Laissue JA, Zimmermann A. 'Neuroendocrine' differentiation in hepatocellular carcinomas (HCCs): immunohistochemical reactivity is related to distinct tumor cell types, but not to tumor grade. Histol Histopathol 1993;8:617-626.

64 Stevens WR, Johnson CD, Stephens DH, et al. Fibrolamellar hepatocellular carcinoma: stage at presentation and results of aggressive surgical management. AJR Am J Roentgenol 1995;164:1153-1158.

65 Vauthey JN, Klimstra D, Franceschi D, et al. Factors affecting long-term outcome after hepatic resection for hepatocellular carcinoma. Am J Surg 1995;169:28-34.

66 Choi SB, Kim KS, Choi JY, et al. The prognosis and survival outcome of intrahepatic cholangiocarcinoma following surgical resection: association of lymph node metastasis and lymph node dissection with survival. Ann Surg Oncol 2009;16:3048-3056.

67 Nakagawa T, Kamiyama T, Kurauchi N, et al. Number of lymph node metastases is a significant prognostic factor in intrahepatic cholangiocarcinoma. World J Surg 2005;29:728-733.

68 Davies JQ, de la Hall PM, Kaschula RO, et al. Hepatoblastoma-evolution of management and outcome and significance of histology of the resected tumor. A 31-year experience with 40 cases. J Pediatr Surg 2004;39:1321-1327. 